Témoigner Témoigner. Entre histoire et mémoire

Getuigen Revue pluridisciplinaire de la Fondation Auschwitz

$124 \mid 2017$

La musique dans les camps

\title{
Trois églises grecques en Afrique centrale : lieux de mémoire russes
}

Drie griekse kerken in Centraal-Afrika: russische Herinneringsplaatsen

\section{Vladimir Ronin}

\section{(2) OpenEdition}

1 Journals

\section{Édition électronique}

URL : https://journals.openedition.org/temoigner/5824

DOI : $10.4000 /$ temoigner.5824

ISSN : 2506-6390

Éditeur :

Éditions du Centre d'études et de documentation Mémoire d'Auschwitz, Éditions Kimé

\section{Édition imprimée}

Date de publication : 2 avril 2017

Pagination : 180-185

ISBN : 978-2-930953-00-7

ISSN : 2031-4183

Référence électronique

Vladimir Ronin, «Trois églises grecques en Afrique centrale : lieux de mémoire russes », Témoigner. Entre histoire et mémoire [En ligne], 124 | 2017, mis en ligne le 30 novembre 2021, consulté le 01

décembre 2021. URL : http://journals.openedition.org/temoigner/5824 ; DOI : https://doi.org/10.4000/ temoigner.5824 


\section{Site mémoriel}

\section{TROIS ÉGLISES GRECQUES EN AFRIQUE CENTRALE : LIEUX DE MÉMOIRE RUSSES}

Entre 1870 et 1970, plus de 700 hommes et femmes originaires de l'empire russe se sont rendus dans les possessions belges d'Afrique centrale. La plupart de ces anciens sujets du tsar ont franchi le pas de

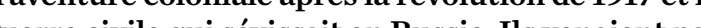
milliers de decifoys 1Afriqu. Ingé hydrographes, géologues, mécaniciens, entrepreneurs, ils ont tous cont lan de maniòre enside lable au Ruappention Ruanda-Uruenon seulement à lepoctue coloniale,

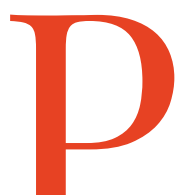

armi les sources qui m’ont permis de reconstituer Ces émigrés russes au cœur de l'Afrique noire (Ronin, 2006; Ronin, 2009), je citerai surtout les listes de copu lato d'État bans les archives de l'ancien ministère des Colos et la presse russe qui paraissait dans les années vingt et trentè Brusses Paris Berlin et Prague..J'.

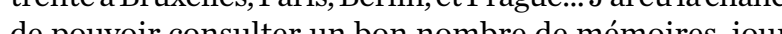

naux intimes et lettres inédits dans 作 coloniaux russes et de réliser, en Begique et en France, une pelite centaine d'interviews d'eux ou de lite centaine Lors denesvoyages dens láp D́s de Dénochict au Rwanda (2015), j'a , au Buru des Russaye dès tant d'anes dées depuis leur dép les résultats de mes recherch les resultats de mes recherches sur le mais non sans intérêt.

Une grande majorité de cesémigrés, très attachés à leur foi et leurs rite chrétiens orthodox, con chrestiens leur vie religieuse. Lours gelis leurs paroisses, leur clengé, its lesses, leurs paroisses, leur clerge, ils les on laissés provisoirement derrière eux, l'Afrique. Dans les grands centres de IAficus La petite diespora russe doxes grecs. industriels du Katansa frécust de temps en tems depris les trente, la très jolie éplise les années l’Ante, la tres jón égise grecque de IAnnonciation a clisabethville (actuelde la fête de Pâques, la plusintontlors

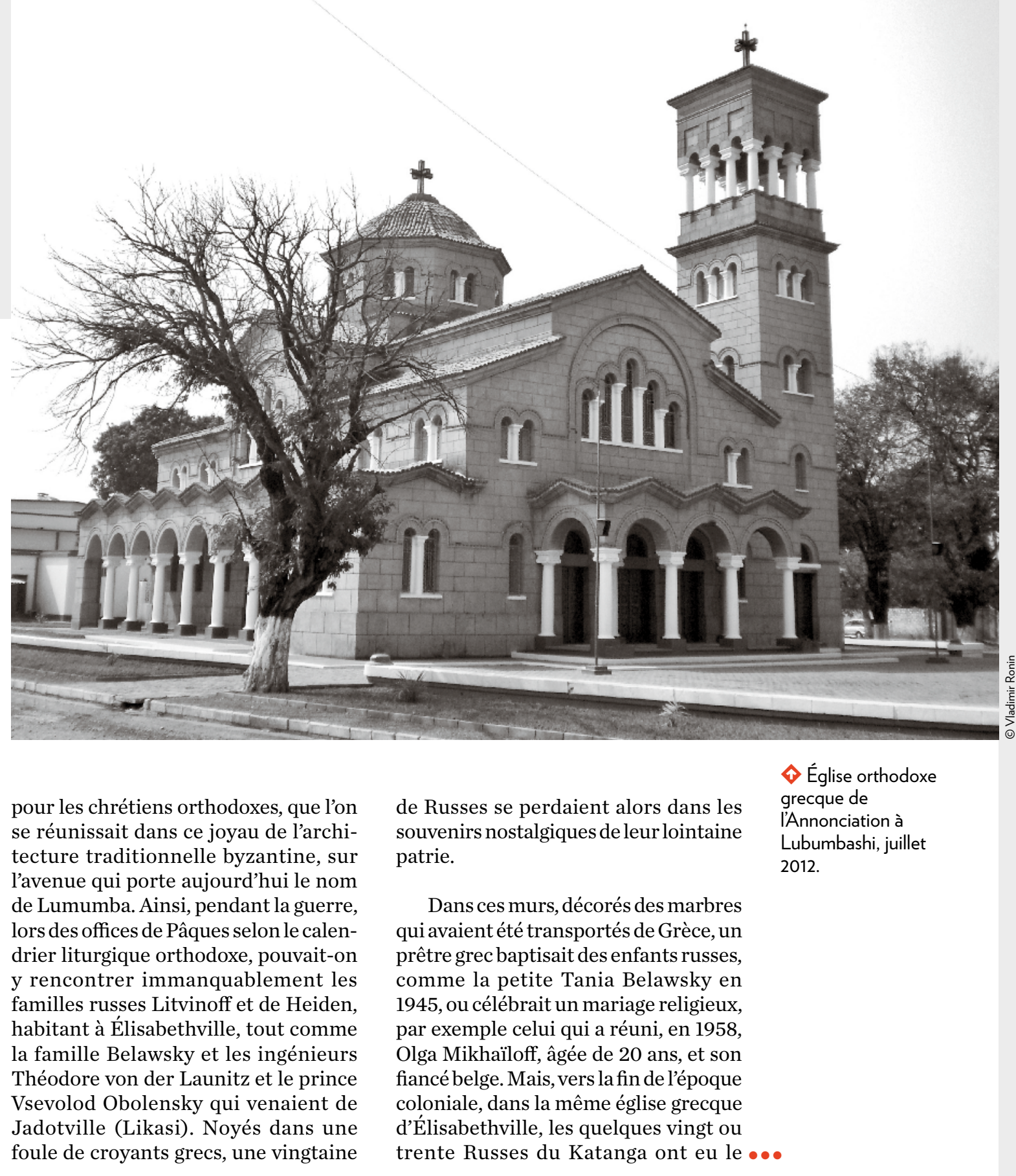


• bonheur d'assister plus d'une fois aux offices célébrès non pas en grec, mais dans leur propre langue liturgique, le slavon. En octobre 1956, un l'́ deltéces avait réuniun burc, estrenud burg, visiter ses compatiotes au Congo belge. d'u avant ce voyage, le patriarche orthodoxe diexandrie, sous lajuridiction duquel se trouvaientes Grecs de toutlecontinent noir, avait des offices dans les églises grecques. églises grecques.

Dans un compte rendu de son voyage, le père Simeon se souvenait de ul'admirable église d'Élisabethville, très haute, à l'acoustique in Elsabethille, très haute, à lacous(Stariko, 1956, p. 16) Avec socold (Starikov, 1956, p.16) Avec soncollegue grec, le prêtre russe y a celébré les vepres et la liturgie dly avice dont

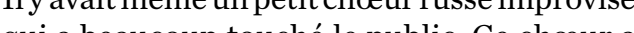
qui a beaucoup touché le public. Ce chœur a éte organisé a loccasion par «unviai connaisseur des chants liturgiques russes », un Belge decrie Il sogissait d'An luin Russie. cou, le chef vétérinaire du «Comité Spécial du Káanga.» Hornis quelques dizaines de Russes d'Élisabethville et de Jadotville, léglise était bondée de centaines de Grecs, enthousiastes etsolidaires, ceque leconpterend soulgnait avec satisfaction. Quelques années apres, le successeur du père Simeon à Johannesburg, larchin fois les Russes dann du du Congo, é 1959 et au prins avant la décolonisation. A Élsabethville, les Grecs ont chaque fois mis a sa disposition leur église pour célebrer les âtices en slavon. Daprèsles mémoires delarchimandrte, lajoie spirituelle de ses compatriotesétait indescripéclise à eux, leurs rites, leur prêtre.p (Cheréglise à eux, leurs rites, leur prêtre...» (Cher-
Mais l'épisode le plus extraordinaire dans la vie des Russes au Katanga belge, un épisode la vie des Russes au Katanga belge, un épisode lie, luinsi, a legise a eu lieu le 6 mai 1945, le jour où les Russe en Europe touchait à sa fin, et même les émiénés rope touchait à sa fin, et mềme les émihaut degré, étaint antisoviétiques au plus haut degre, étaient, depuis Stalingrad, fortepeuple sur l'Allemegne les victoires de leur peuple sur lAllenagne. Aussi etaient-ils très positivenent inpressionses par les quelques pire de Staline au cours de la pire de Stahthe au cours de la guerre, à savoir la testauration de certaines tradions de lépoque bienveillans le Stalinge, lattention presque doxe russe, et le fait que la propage orthodoxe russe, et le fart que la propagande soviètique soit passée des hymnes aux "proletaires de la nation russe le $^{\text {er }}$ jes a «la gra de la nation russe». Le 1 janvier 1944,I'Tnternationale, nutiqué soviétique, a céde la place à un chant patriorépubliques libres, forgé pour toujours par reppubliques libres, forgee pour toujours par la
Grande Russie.»

Après la liturgie de Pâques de 1945 lorsqu'un groupe de Russes sortit de l'église grecque delisabethville, lun deux, Theodore von der Launitz, leur fait signe de s'arrêter. dont le pire, le maire de Sint-Péterborste dont le pere, le maire de Saint-Pétersbourg riste révolutionne is dit avec in Histe revolutionnaire, il dit a ce insistance: russe ! Et voil̀̀ donc une scène pre hyne russe!» Et voilà donc une scène presque délil'A qui s'est jouée sur le parvis de l'eglise de et de fils d'anci.. Ungroupe danciens officiers et de fils danciens officiers, après avoir comcivile longue et implacable, aprs a civile longue et inplacable, après avoir hai pendant plus de vingt ans tout ce qui sentait

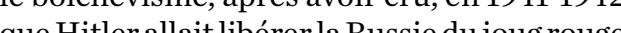
que Hitler allait libérer la Russie dujoug rouge,
Pâques, au centre d'une ville coloniale belge, l'hymne national de l'Union belge, l hym national de lUnion soviette scie. Un déni-siecle plus tard cettescène ma été racontée par un des participants, lepté

Un autre lieu en Afrique centrale, qui a vu passer nombre d'émigrés russe engagés pour l’ouvre coloniale belge, est Bujumbura, la capitale du Burund Congo, le so juin 1960, a été suivie, on ne le sait que trop bien, par de graves La proclamation de lindépendance du

lence, les coloniaux, dont ceux d'origine usse, fuient en masse vers l'Europe ou, de l'Et du Congo au Rus un territoire sous tutelle de l'ONU et en 1960 encore sous l'autoríc admiistrative belse Une partie de la comrist dans les a che chef-lieudu Ruanda-Unindi,Usombura (Bujumbura).

Jusquà l'indépendance du Rwanda (1962), Iingenieur agro-

$\diamond$ Église orthodoxe grecque à

Bujumbura,

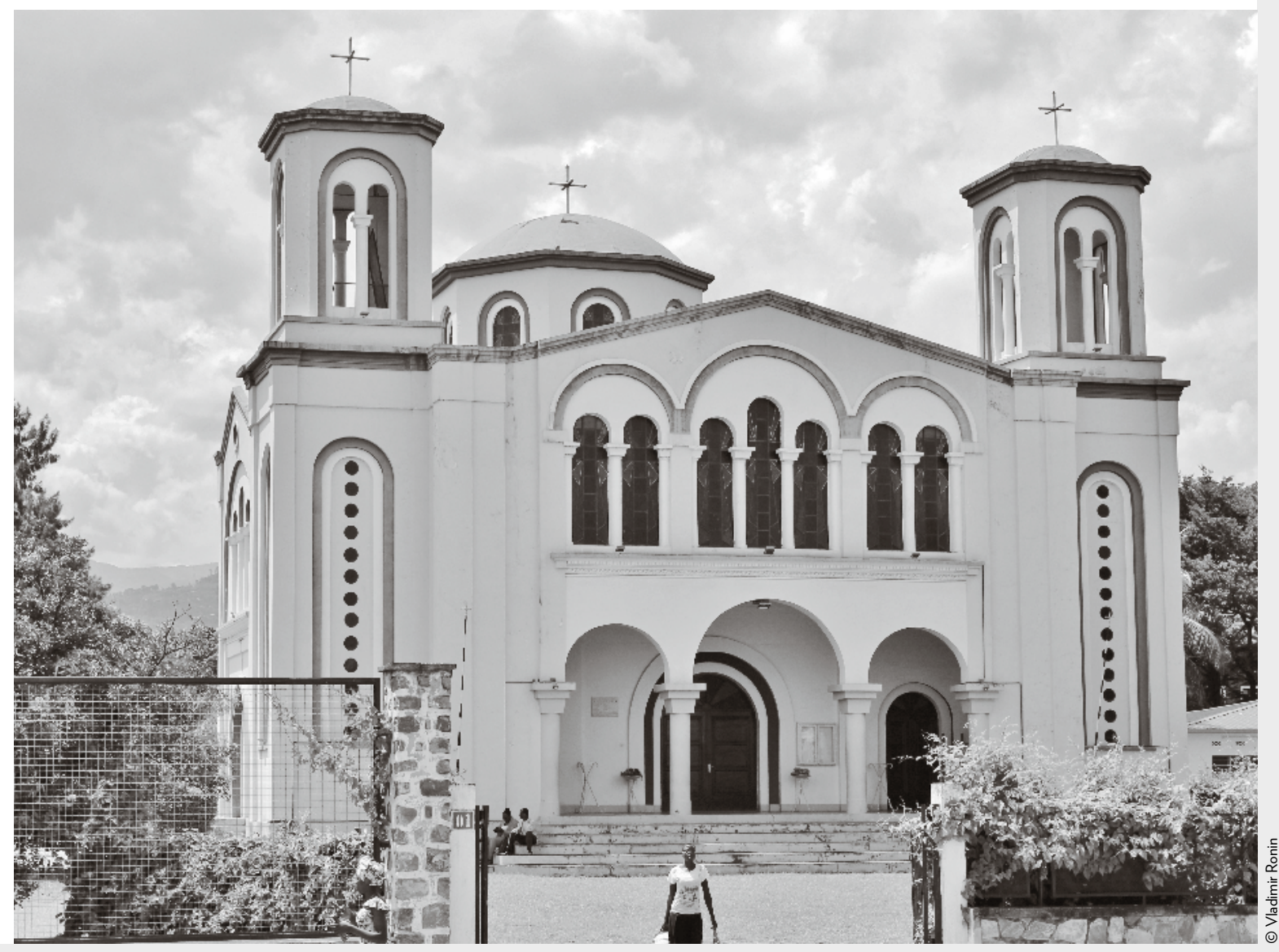


œ. navette entre Usumbura, alors relativement calme, et sa station de recherches agronomiques près de Bukavu. C'est dans les lettres adressées à sa femme, à Bruxelles, que nous con samedi sir 8 avil, tandis que d’utres Européens soir 8 ancill, tandis que dautres Européns sont allés von un nonveau film avec Brigitte Bardot en vedette, une dizaine de Russes in sont rendus aux vigiles dans leglise grecque, haugue auparimi Bujuara, elle fut construte entre 1955 et Bujumbura, elle fut construite entre 1955 et 1959. A en croire Kutcharof, lui et ses compatrotes sont restés fort mécontents de la façon dont les Grecs ont célébré les offices de Pâques cette annéclà : «Une farce, voilà ce que c'est. comprendre quique ce soit. comprendre quoi que ce soit.

Toutlemonden’apasquittéle Congoen 1960 Plusieurs ex-coloniaux russes n’avaient pratiPlusieurs ex-coloniaux russes navaient pratiquement aucun lien en Europe pas de famille, pas de maison, pas dhèritages à attendre... Où de 25,30 ou mê 40 ans pasśs de 25,30 ou nêne 40 ans passés en Afrque? DansleCongoindé russes ót à Kolwezi, un des cences les plus inportan les plus importants du pays, le conte Vladimir privéde, ingenieur deśr. fils privé de la dernièreimpératrice de Russie, était, en 1964 et jusquen 1970 lereprentant officiel 1966, la Ǵcan 1966, la Gécanines). Une figure clé Kolwezi.I portait pratiquin senl la responsabitite de toute la populationlocale: environ 5000 Européens et 500 Congolais. Apraxine s'est assez bien acapté au nouvel ordre des choses après la décolonisation. "Très slave, très grand seigneur» selon lexpression dun écrivain belge en visite au Katanga (Marchal, 1969, p. 13), cet aristocrate russe jouissait de lautorite naturelle aussi bien dans les mines et les usines que dan
Parmi les affaires dont il devait s'occuper pour le bien-être de la population de Kolwezi, il y en avait une qu'il prenait personnellement 列 grecque. Même si l'église Saint-Georges n’a été achevée qu'en 1974, quatre ans après le départ du «bwana Apraxine» du Congo, la communauté grecque de l'époque lui devait beaucoup

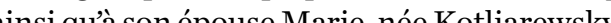
Cen'est pas pour rien quil , nee Kotliarewsky.

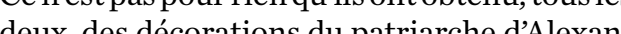
drie drie. La contesse Marie Apraxine me racont plus tard qu'elle avait aussi offert à l'eglise en avaient apportées en Afrique.

Un demi-siècle, c'est beaucoup de temps et lorsqu'en juillet 2012, j'ai franchi le seuil de l'église Sa juillet 2012, jai franchi le seuil de presque ain de Gres das la ville. Quant aux presque pas de Grecs dans la ville. Quant aux parsîn conglais, de contession orthodoxent Apraxine ni d'une icône russe dans leur église. Apraxine ni d'une icône russe dans leur église. du Katana, Meletios, qui ma tementindić tement indiqué une petite icone représentant Ran Radone, Cette belle petite icôn-Trité près de Moscou. é émouvant de la présence rouvenir unique et énouvant de la présence russe en Afrique D’ailleurs Mor Meletion m’o eussiparlébrièveDaint lui avaient rendu visite à Kolwezi. Ils éta.

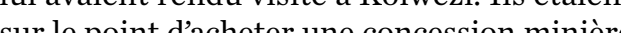
sur le point dacheter une concession minière au Katanga et ont prié leveque grec de veni Russes au Congo n'est peut-être pas en Russes a Coéc Concore

Vladimir Ronin KU Leuven - Campus Antwerpen

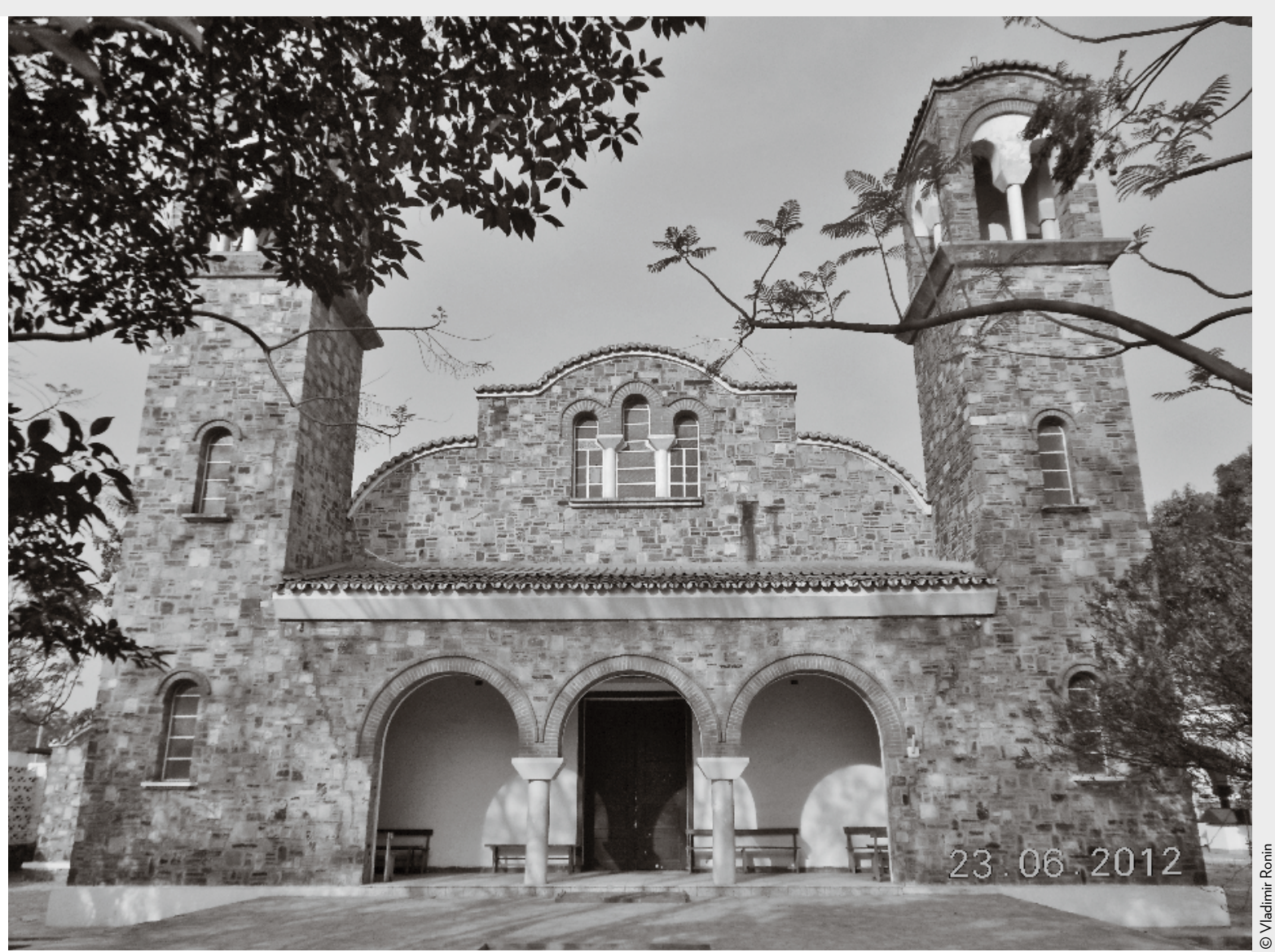

¿ Église orthodoxe grecque St-Georges à
Kolwezi, juin 2012.

\section{Bibliographie}

$\diamond$ Chernay Alexey (1981) : Russian Odyssey. The Life Path of an Exile, Roodepoot,

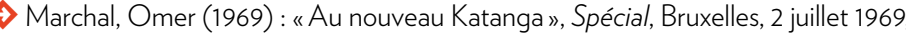

$\diamond$ Ronin, Vladimir (2006): « Les Russes au Congo belge », Contactforum 23 november 2005, Brussel, Koninklijke Vlaamse Academie voor Wetenschappen en

$\Rightarrow$ Ronin, Vladimir (2009): « Russkoe Kongo », 1870-1970 [«Le Congo russe », 1870-

$\diamond$ Starikov, Simeon (1956): « Missionerskaja poezdka vglub' Afriki» [Un voyage
missionnaire au coeur de l'Afrique], Pravoslavnaja Rus', Jordanville (N.Y.'), 24, p. 15-17. 